\title{
BS26
}

\section{Broadband Seismic by Means of Dispersed Source Arrays}

\author{
M. Caporal* (Delft University of Technology) \& G. Blacquière (Delft \\ University of Technology)
}

\section{SUMMARY}

Seismic sources deployed during a particular survey are historically chosen to be equal. However, from a physical point of view the constraint of using only identical source units is not required. We suggest to abandon this constraint and to replace, or reinforce, traditional broadband sources with narrower-band devices, together representing a Dispersed Source Array (DSA). Operational flexibility and acoustic energy transmission efficiency are expected to considerably improve. In addition, the DSA concept opens the possibility to choose tow depths and spatial sampling intervals that are optimum for specific sources (bandwidths). The ensemble of sources incorporated in the array is designed to cover the entire temporal and spatial bandwidth of interest. 


\section{Introduction}

Particularly in the last decade, broadband seismic acquisition has become a very important topic both for academia and the industry and the potential benefits for a geographical region such as the Middle East are significant. It is indeed widely accepted that the contribution of both high and low frequencies is of fundamental importance to guarantee high-quality seismic imaging. On the one hand, high frequencies provide sharper wavelets resulting in an improved vertical resolution, where with resolution we mean the ability to distinguish two different features from one another. On the other hand, low frequencies drastically reduce the wavelet sidelobes and therefore the potential interference among neighbouring seismic events. They also translate in better signal penetration, suffering less from scattering and attenuation. Furthermore, they play a crucial role in seismic inversion for velocity and impedance models.

However, the most commonly used seismic sources, both on land and offshore, produce relatively little energy for what concern the very low and very high frequencies of seismic interest. In fact, from a practical point of view, a significant effort is required to profitably produce and operate source units that are adequately efficient in the whole desired frequency range. Enhancing the system efficiency in the range limits is indeed a very active field of research. A vast literature production can be found, among which: Moldoveanu et al. (2006), Bagaini (2008), Baeten et al. (2010), Soubaras and Dowle (2010) and Landro and Amundsen (2014).

Following the guidelines drawn by Berkhout (2012), we propose to employ more than one type of source during the same seismic survey, together representing a disperse source array (DSA). Each source unit involved in the acquisition scheme is dedicated to a narrow frequency bandwidth, without the need to satisfy the wideband requirement. The ensemble of sources incorporated in the array is designed to cover the entire temporal and spatial bandwidth of interest.

\section{Method}

To illustrate and test the DSA concept, four different source unit types are numerically simulated: ultralow-, low-, mid- and high-frequency sources with band intervals 2-6, 5-15, 10-30 and 20-60 Hz, respectively. Note that each source among the selected ones spans a frequency bandwidth corresponding to the same number of octaves. Given bandwidths are partially overlapping. In the following examples, such sources are designed to guarantee optimal amplitude conditions in the whole frequency band of interest when combined together. In Figure 1a the amplitude spectra of aforementioned sources are shown.

Such source units could ensure, each one within its specific frequency band, a more efficient acoustic energy transmission than today's broadband alternatives. The emission properties of each device can, in fact, be separately optimized. Modern multiple-driver loudspeaker systems are based on the same key concept and their improved performances are demonstrated (Borwick, 2001).

In regard to the source sampling, according to the Shannon-Nyquist sampling theorem, the source interval should be smaller than a half period of the smallest horizontal wavelength. Thus, the required number of low-frequency source units (shots) would be lower than the required number of highfrequency source units (shots).

Furthermore, the performances of such sources may also significantly improve from an operational flexibility point of view. Thanks to this, the DSA concept is particularly suited to blended acquisition and simultaneous shooting. In fact, the overlap among the frequency bands of the different sources is limited or, in the extreme case, inexistent.

In addition for the marine surveys, to reduce the effect of the source ghosts, each source unit can be positioned at the optimum specific depth level below the water surface. Choosing tow depths equal to 
a quarter of the central wave length of the device $\left(z_{s}=\lambda / 4\right)$, ghost destructive interference can be largely avoided.

The employment of narrowband or monochromatic low-frequency sources in seismic acquisition has also been proposed by Dillinger et al. (2012), mainly as a reinforcement for conventional broadband acquisition. On the other hand, an interesting first attempt of DSA real data acquisition and inversion (FWI-based) has been carried on and presented by Kim and Tsingas (2014).

In the following, a preliminary example of DSA synthetic data migration is provided. The reader is also referred to Caporal et al. (2015, submitted) for a more detailed theoretical explanation of the method.

\section{Example}

The reflectivity subsurface model we used is shown in Figure 1b. The subsurface is featured by a horizontal pack of layers, representing the target area, and a salt structure that serves as a strong internal multiples generator. Two different datasets corresponding respectively to a conventional broadband survey $(2-60 \mathrm{~Hz}$ sources) and a DSA survey have been simulated with a finite difference algorithm. Free-surface boundary condition has been applied. The migration outputs are shown respectively in Figure 1c and Figure 1d. As expected, the quality of the DSA migration result is analogous to the quality of the broadband migration result. Migration has been performed by means of an FWM (Full-Wavefield Migration) algorithm. The reader is referred to Berkhout (2014) and Davydenko and Verschuur (2014) for further details.

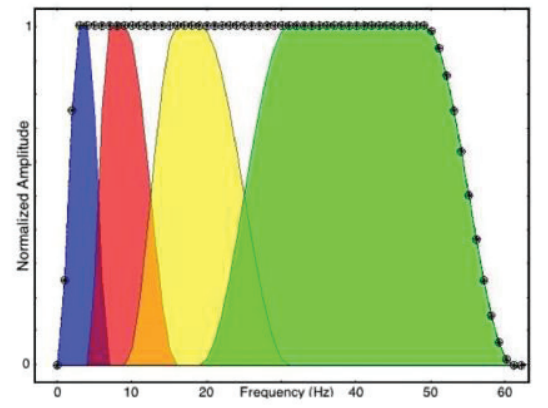

(a)

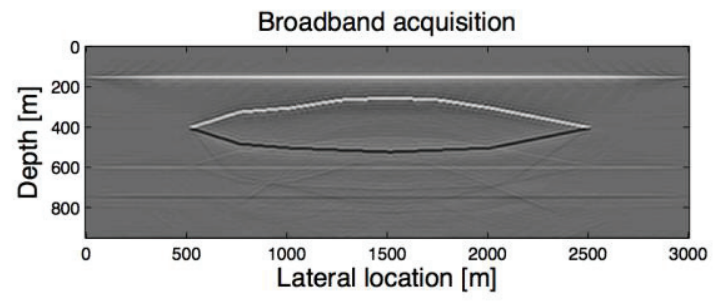

(c)

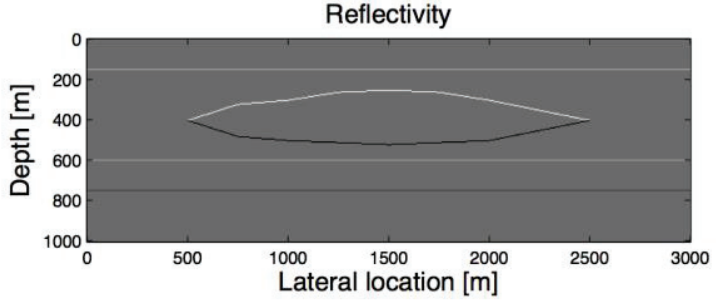

(b)

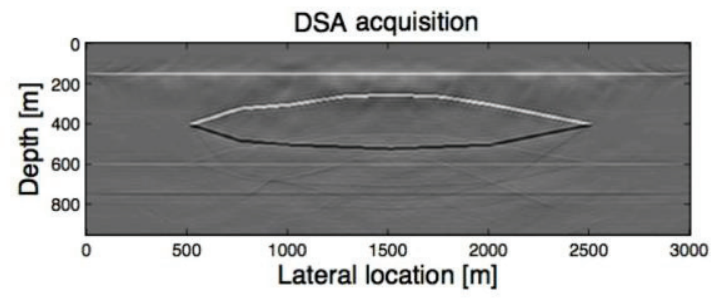

(d)

Figure 1 (a) Amplitude spectra correspondent to chosen ideal source units. (b) Reflectivity model used for generating reflection data for the simulations is presented. Migration output are shown: (c) broadband acquisition, (d) DSA acquisition. 


\section{Conclusions}

Applying the DSA concept, it is possible to make some fundamental improvements by changing the system architecture. The design of narrowband sources, which together cover the total bandwidth of interest, may become significantly simpler. Additionally, the source density could be chosen in a frequency dependent manner. Applying this concept, surveys may be carried out by acquisition systems that are considerably less complex and more flexible than the ones that are used today. Furthermore, the possibility to use tow depths that are optimum for the specific central frequency of narrowband sources gives extra benefits if we look at the source ghost issue.

Ghost destructive interference and low frequency attenuation due to shallow tow depths are largely avoided. Interesting and encouraging preliminary migration outputs from DSA datasets have already been produced. Furthermore, considering the potential significant flexibility the DSA concept would give to the acquisition system, the design of an optimum blended survey design is a logical ne xt step forward in this framework.

\section{Acknowledgements}

The authors would like to thank the sponsors of the Delphi consortium for the simulating discussions during the Delphi meetings and their continuing financial support.

\section{References}

Baeten, G. J. M., A. Egreteau, J. Gibson, F. Lin, P. Maxwell, J. J. Sallas [2010] Lowfrequency generation using seismic vibrators: 72th Conference and Exhibition, EAGE, Expanded Abstracts.

Bagaini, C. [2008] Low-frequency vibroseis data with maximum displacement sweeps: The Leading Edge, 27, 582-591.

Berkhout, A. J. [2012] Blended acquisition with dispersed source arrays: Geophysics, 77 (4), A19-A23.

Berkhout, A. J. [2014] Review paper: An outlook on the future seismic imaging, part II: Full-wavefield migration: Geophysical Prospecting, 62 (5), 931—949.

Borwick, J. [2001] Loudspeaker and Headphone Handbook: Focal Press.

Caporal, M., G. Blacquière, A. J. Berkhout [2015] (submitted), Seismic acquisition with Dispersed Source Array: first results: 85th Annual International Meeting, SEG, Expanded Abstracts.

Davydenko, M., and D. J. Verschuur [2014] Full wavefield migration in three dimensions: 84th Annual International Meeting, SEG, Expanded Abstracts, 3935-3940.

Dillinger, J. A., J. T. Etgen, and G. Openshaw [2012] Seismic acquisition using narrowband seismic sources: US Patents 2012/0155217 A1.

Kim, Y., and C. Tsingas [2014] Land full waveform inversion for dispersed source arrays: 76th Conference and Exhibition, EAGE, Expanded Abstracts.

Landro, M., and L. Amundsen [2014] Maximizing the ultra-low frequency output from air guns: 76th Conference and Exhibition, EAGE, Expanded Abstracts.

Moldoveanu, N., L. Combee, M. Egan, W. Abriel, and G. Hampson [ 2006] Over/under towed-streamer acquisition: A method to extend the seismic bandwidth to both higher and lower frequencies: 76th Annual International Meeting, SEG, Expanded Abstracts, 10-15.

Soubaras, R., and R. Dowle [2010] Variable-depth streamer - A broadband marine solution: First Break, 28, 89-96. 